\title{
Adsorption on a periodically corrugated substrate
}

\author{
K.Rejmer and M.Napiórkowski \\ Instytut Fizyki Teoretycznej, Uniwersytet Warszawski \\ 00681 Warszawa, Hoża 69, Poland
}

November 12, 2018

\begin{abstract}
Abstract. Mean field analysis of the effective interfacial Hamiltonian shows that with increasing temperature the adsorption on a periodically corrugated substrate can proceed in two steps: first, there is the filling transition in which the depressions of the substrate become partially or completely filled; then there is the wetting transition at which the substrate as a whole becomes covered with a macroscopically thick wetting layer. The actual order and location of both transitions are related to the wetting properties of the corresponding planar substrate and to the form of corrugation. Certain morphological properties of the liquid-vapor interface in the case of a saw-like corrugated substrate are discussed analytically.
\end{abstract}

PACS numbers : 68.45.Gd, 68.35.Md, 68.35.Rh 


\section{Introduction}

Although the wetting of homogeneous and planar substrates is nowadays a relatively well understood phenomenon [1-3] its counterpart corresponding to the adsorption on corrugated substrates still poses many questions which remain unanswered in spite of much recent experimental and theoretical work [4-19] devoted to these systems. Different apects of wetting on a corrugated substrate, e.g. the order of the wetting transition, the location of the wetting point on the thermodynamic phase diagram, and the structure of the emerging liquid-vapor interface have been usually refered to the wetting properties of the corresponding planar substrate which indeed serves as the natural reference system. However, recent work on the special type of non-planar substrate the infinitely extended wedge-like substrate [20-23] - points at a novel type of transition characteristic for this system. This is so-called filling transition which - in our opinion - becomes also relevant in the case of adsorption on a periodically corrugated substrate. It takes place at the bulk liquid-vapor coexistence and at a temperature that is lower than the wetting temperature of the corresponding planar substrate. In the course of the filling transition the width od the wetting film becomes macroscopically thick in the center of the wedge and it remains thin far away from the wedge center; there the interface locally interacts with the planar substrate only which at the filling transition remains nonwet.

Our mean-field analysis of adsorption on a periodically corrugated substrate aims at showing that the filling transition mentioned above can be also relevant in this case and precedes the wetting transition [19]. Thus two kinds of transitions, i.e. the filling and the wetting transition can be present in the scenario of adsorption on the corrugated substrate.

In Chapter II we introduce the system and present the thermodynamic analysis of the problem. It shows which properties of the substrate's shape are relevant for the filling transition. We also comment on the phenomenological Wenzel's law $[24,25]$ locating the wetting temperature for a corrugated substrate. Chapter III contains the analysis of the adsorption in the case when the corrugation of the substrate has the special saw-like form. This analysis is based on rather general considerations using the concept of the effective interfacial Hamiltonian. They allow us to extract information not only about the order of the filling and the wetting transitions but also point at certain structural properties of the liquid-vapor interface. Chapter IV contains analytic discussion of the above issues. In this case the shape of the interface can be obtained explicitely and its scaling properties in different temperature regimes can be transparently presented. In Chapter V we summarize our results.

\section{Thermodynamic description}

We consider a substrate which is periodically corrugated in the $\mathrm{x}$-direction 
and translationally invariant in the y-direction. It's shape is described by the function $z=b(x)$ with period $2 a$, i.e. $b(-a)=b(a)$. Moreover, we assume that $b(-x)=b(x)$ and that $b(x)$ is monotonically increasing for $0<x<a$; $b(x)$ has the minimum at $x=0$ and the maximum at $x=a$. The space above the substrate is occupied by the inhomogeneous fluid and the thermodynamic conditions are chosen such that for $z \rightarrow \infty$ the bulk vapor is the stable thermodynamic phase. Close to the substrate, due to its preference for a liquid phase a liquidlike layer is formed which separates the vapor from the substrate. The knowledge of the shape of the liquid-vapor interface $z=f(x)$ and the width of the liquid-like layer $l(x)=f(x)-b(x)$ as function of the thermodynamic state of the system allows one to discuss the possible filling and the wetting transition taking place in this system. We do not put any constraints on the amount of liquid adsorbed on the substrate. Such contraints might lead to the interfacial configurations which break the substrate symmetry [26]; this is not the case in our analysis.

In this Chapter we analyse the problem thermodynamically by considering macroscopic configurations of the interface and evaluating the corresponding surface free energies. In order to make the notation consistent with that employed later within the effective Hamiltonian approach (Chapters III and IV) we denote the vapor phase and the liquid phase as phases $\alpha$ and $\beta$, respectively.

We assume that the substrate imposes its periodicity on the allowed interfacial configurations and thus our analysis is reduced to a single subtrate's depression extending on the segment $[-a, a]$. Moreover, due to the substrate's symmetry $b(x)=b(-x)$ it is enough to concentrate on the segment $[0, a]$. One takes into account two types of competing interfacial configurations: the first corresponds to the partial filling of the depression, Fig.1a, and the second corresponds to the interface located above or exactly at the top of the substrate, i.e. $f(x) \geq b(a)$, see Fig.1b. The free energy of the depression filled completely with the phase $\alpha$ serves as the reference point. Then the excess surface free energies corresponding to the above two cases have the following form

$$
\begin{gathered}
\Delta F_{1}\left(x_{1}\right)=2 x_{1} \sigma_{\alpha \beta}+L\left(x_{1}\right)\left(\sigma_{w \beta}-\sigma_{w \alpha}\right)= \\
=\sigma_{\alpha \beta}\left[2 x_{1}-L\left(x_{1}\right) \cos \theta\right], \\
\Delta F_{2}=\sigma_{\alpha \beta}[2 a-L(a) \cos \theta],
\end{gathered}
$$

respectively. $x_{1}$ denotes the value of the abscissa at which the planar segment of the $\alpha$ - $\beta$ interface makes the contact with the substrate, i.e. $f\left(x_{1}\right)=b\left(x_{1}\right) . \sigma_{\alpha \beta}$, $\sigma_{w \alpha}, \sigma_{w \beta}$ are the $\alpha-\beta$, substrate-phase $\alpha$, substrate-phase $\beta$ surface tensions, and

$$
L\left(x_{1}\right)=\int_{-x_{1}}^{x_{1}} d x \sqrt{1+b_{x}^{2}(x)}
$$


is the length of the substrate-phase $\beta$ interface. In Eqs.(2.1-2.2) the Young equation has been used and $\theta$ denotes the planar substrate contact angle.

The equilibrium interfacial configuration corresponds to the value $\bar{x}_{1}$ of the contact point abscissa which minimizes the excess free energy. It solves the following equation

$$
\begin{array}{r}
0=\Delta F_{1, x}\left(\bar{x}_{1}\right)=2 \sigma_{\alpha \beta}\left(1-\sqrt{1+b_{x}^{2}\left(\bar{x}_{1}\right)} \cos \theta\right)= \\
2 \sigma_{\alpha \beta}\left(1-\frac{\cos \theta}{\cos \psi\left(\bar{x}_{1}\right)}\right),
\end{array}
$$

where $\psi\left(\bar{x}_{1}\right)$ denotes the actual contact angle on the corrugated substrate shown in Fig.1a. Thus the surface free energy has an extremum for such value of $\bar{x}_{1}$ that the corresponding contact angle $\psi\left(\bar{x}_{1}\right)$ becomes equal to the contact angle $\theta$ on the planar substrate. The actual minimum is located by inspecting the second derivative of the excess free energy

$$
\Delta F_{1, x x}\left(\bar{x}_{1}\right)=-2 \sigma_{\alpha \beta} \frac{b_{x}\left(\bar{x}_{1}\right) b_{x x}\left(\bar{x}_{1}\right)}{\sqrt{1+b_{x}^{2}\left(\bar{x}_{1}\right)}} \cos \theta .
$$

For substrates under consideration one has $b_{x}>0$ for $0<x<a$ and the sign of $\Delta F_{1, x x}\left(\bar{x}_{1}\right)$ is determined by the sign of $b_{x x}\left(\bar{x}_{1}\right)$, i.e. by the curvature of the substrate. $\bar{x}_{1}$ corresponds to the excess free energy minimum if the substrate is convex at the corresponding point $\bar{x}_{1}$ (i.e. $b_{x x}\left(\bar{x}_{1}\right)<0$ and $b(x)$ is concave at $\bar{x}_{1}$ ). On the other hand if the substrate is concave at $\bar{x}_{1}$ (i.e. $b_{x x}\left(\bar{x}_{1}\right)>0$ ) then the excess free energy has maximum. For an arbitrary substrate shape $b(x)$ with several inflection points there may be many competing equilibrium states leading to a rather complicated phase diagram.

For periodic substrates with a single inflection point $x_{i n f} \in[0, a]$ the situation is more transparent. An example of such a substrate is given by $b(x)=$ $a(1-\cos (x \pi / a))$ for which one has $b_{x}(0)=b_{x}(a)=0$ and $x_{i n f}=a / 2$. For appropriate range of values of the contact angle $\theta$ the excess free energy has both maximum and minimum located at $x_{\max }$ and $x_{\min }$, respectively. Upon increasing the temperature $\theta$ decreases, $x_{\max }$ decreases, and $x_{\min }$ increases. The limiting value $\theta_{0}$ such that for $\theta<\theta_{0}$ these two extrema of the excess free energy exist is determined by the shape of the substrate: $\theta_{0}=\psi\left(x_{\text {inf }}\right)$. For temperatures below $T_{0}$ such that $\theta\left(T_{0}\right)=\theta_{0}$ the depression remains filled with the bulk phase $\alpha$. This case is called the empty depression and $\bar{x}_{1}=0$. For $T>T_{0}$ the depression may be partially filled with (unstable in the bulk) phase $\beta ; \bar{x}_{1}=x_{\min } \in\left[x_{i n f}, a\right]$. The equilibrium configuration is selected by the excess free energy balance, Fig.2. The transition from the empty to the partially filled configuration which takes place at $T_{f}$ is first-order. The equilibrium value $\bar{x}_{1}$ changes discontinuously from 0 to $\bar{x}_{1 f}$ which is the smallest value of $x_{\min }$ such that $\Delta F_{1}\left(\bar{x}_{1 f}\right) \leq 0$. We call it the filling transition. The corresponding value 
$\theta_{f}$ of the contact angle is given by

$$
\cos \theta_{f}=\frac{2 \bar{x}_{1 f}}{L\left(\bar{x}_{1 f}\right)} .
$$

Upon further increase of the temperature $x_{\min }$ increases towards $a$; this limiting value of $\bar{x}_{1}$ at which the whole depression is filled with the phase $\beta$ is achieved for $\theta=0$, i.e. at the planar substrate wetting temperature $T_{w}$. Still further increase of the temperature does not change the excess free energy balance and the whole substrate remains covered by the $\beta$-like layer of growing width. However, the analysis of this further growth is beyond the present thermodynamic description.

The above analysis shows that the very existence of a nontrivial filling transition depends substantially on the substrate's shape. If each depression is strictly concave (i.e. $\left.b_{x x}(x)>0, x \in(-a, a)\right)$ then the thermodynamic argument points at non-existence of the filling transition leading to a partially filled depression. For example, when the periodic piecewise concave substrate consists of the arcs of circles of radius $R$ then

$$
\Delta F\left(x_{1}\right)=2 R \sigma_{\alpha \beta}\left[x_{1} / R-\arcsin \left(x_{1} / R\right) \cos \theta\right],
$$

see Fig.3. Upon increasing the temperature the depression remains empty until the contact angle reaches the value $\theta_{f}$ such that $\cos \theta_{f}=\sin \delta / \delta$ at which the whole depression becomes filled with the $\beta$-phase and $\bar{x}_{1}=a$. In this case the circle's arc forming the depression is tangent to the $x$-axis at $x=0$ and $\pi-2 \delta$

denotes the angle between the two arcs meeting at $x=a$. On the contrary, for piecewise strictly convex substrate (i.e. $b_{x x}(x)<0$ for $x \in[-a, 0)$ and $\left.x \in(0, a]\right)$ the filling of the depression starts at its center and proceeds continuously upon increasing the temperature up to the point $x_{1}=a$. For example, when the periodic piecewise convex substrate consists of the arcs of circles of radius $R$ then

$$
\Delta F\left(x_{1}\right)=2 R \sigma_{\alpha \beta}\left[x_{1} / R-\left(\delta-\arcsin \left(\sin \delta-x_{1} / R\right)\right) \cos \theta\right],
$$

see Fig.4. In this case the tangent to each of the two circle arcs forming the depression at $x \in[-a, a]$ is horizontal at $x= \pm a$ and $\pi-2 \delta$ denotes the angle between the two arcs meeting at $x=0$.

In this case the depression becomes completely filled at $\theta=0$, i.e. at $T=T_{w}$.

The above macroscopic discussion also sheds light on the phenomenological Wenzel's criterion $[24,25]$ locating the wetting temperature $T_{r}$ for a rough substrate. Accordingly, if one compares two interfacial configurations corresponding to the depression either completely filled with the $\alpha$-phase (the so-called empty depression) or completely filled with the $\beta$-phase then equating their free energies leads to the following equation for $T_{r}$

$$
\cos \theta\left(T_{r}\right)=\frac{2 a}{L(a)} .
$$


The inverse of the r.h.s. of the above equation is the so-called roughness factor which measures the ratio of the actual corrugated surface area to its projection on the plane. Eq.(2.9) determines - according to the Wenzel's criterion - the wetting temperature of the rough substrate as compared to the planar substrate case for which $\theta\left(T_{w}\right)=0$. However, this phenomelogical criterion disregards the partially filled configurations and the transitions leading to them which may actually preempty the transition to a completely filled depression. In addition, this criterion does not distinguish transitions to the completely filled depression and those to the state in which the interface is removed macroscopically far away from the substrate as a whole. On the other hand if one looks at the filling transition for an infinitely extended wedge - which is the special limiting case of the substrates we consider here - then the filling transition temperature is correctly given by the Wenzel's rule. The same holds true for the special kind of concave substrate whose free energy is described by Eqs.(2.7).

In the case of the periodic saw-like substrate, i.e. $b(x)=|x| \cot \varphi$, where $\varphi$ denotes half of the saw opening angle the above thermodynamic arguments point at the problems which need to be resolved at the more microscopic level. The excess free energy

$$
\Delta F\left(x_{1}\right)=2 \sigma_{\alpha \beta} x_{1}(1-\cos \theta / \sin \varphi)
$$

is linear function of $x_{1}$. The positive values of the coefficient $(1-\cos \theta / \sin \varphi)$ correspond to the empty depression and the negative values to the completely filled case. At the transition temperature $T_{\varphi}$ such that $\cos \theta\left(T_{\varphi}\right)=\sin \varphi$ no $\bar{x}_{1^{-}}$ value is distinguished by the present macroscopic argument. This corresponds to a degenerate case in which each configuration with $\bar{x}_{1} \in[0, a]$ has the same value of the surface free energy. One certainly needs a more microscopic approach to discuss this case and to distinguish between the different interfacial configurations with the same surface energy. This approach, in addition to the surface contributions to the free energy should involve the analysis of the line contributions as well. The next chapter is devoted to this problem.

\section{The interfacial configurations}

In this chapter we analyze the interfacial configurations in the presence of the periodic saw-shaped substrate introduced above. We restrict our analysis to a single section $x \in[-a, a]$ in which the substrate is described by $z=b(x)=|x| \cot \varphi$. Our mean-field approach is based on the effective Hamiltonian description which has been rather succesfully employed in discussing various interfacial problems [2,3,15-19,22,23].

\section{IIIa. The effective Hamiltonian}


The effective Hamiltonian has the following form

$$
\mathcal{H}[f]=\int_{-a}^{a} d x\left\{\frac{\sigma_{\alpha \beta}}{2}\left[\left(\frac{d f(x)}{d x}\right)^{2}-\cot ^{2} \varphi\right]+\frac{V(l(x))}{\sin \varphi}\right\},
$$

where the film thickness $l(x)=f(x)-|x| \cot \varphi$ is measured along the z-axis. The form of the effective potential $V(l)$ which desribes the interaction of the interface with the substrate [1-3,15-19,22] will be specified later depending on the order of the wetting transition on the planar substrate that we want to refer to. The above form of the effective Hamiltonian is valid for not too rough substrate, i.e. for $\varphi$ close to $\pi / 2$ or $\cos ^{2} \varphi \ll 1$.

The equilibrium interfacial configuration $\bar{f}$ minimizes $\mathcal{H}[f]$ and solves the equation

$$
\sin \varphi \sigma_{\alpha \beta} \frac{d^{2} \bar{f}(x)}{d x^{2}}=V^{\prime}(\bar{l}(x))
$$

supplemented by the boundary conditions $\bar{f}^{\prime}(0)=\bar{f}^{\prime}(a)=0$. (As before, due to the symmetry of the problem we restrict our analysis to the segment $[0, a]$.) Integrating Eq.(3.2) one obtains

$$
\frac{\sigma_{\alpha \beta}}{2} \sin \varphi\left[\left(\frac{d \bar{l}}{d x}\right)^{2}-\left.\left(\frac{d \bar{l}}{d x}\right)^{2}\right|_{x=0}\right]=V(\bar{l}(x))-V_{1},
$$

where $\bar{l}_{1}=\bar{l}(0)$ and $V_{1}=V\left(\bar{l}_{1}\right)$. Since $\bar{l}^{\prime}\left(0^{+}\right)=\bar{l}^{\prime}\left(a^{-}\right)=-\cot \varphi$ it follows from Eq. (3.3) that $V\left(\bar{l}_{1}\right)=V\left(\bar{l}_{2}\right)=V_{1}$, where $\bar{l}_{2}=\bar{l}(a)$.

The equilibrium solution of Eq.(3.3) $\bar{l}(x)$ fulfills the constraint

$$
a=\sqrt{\frac{\sin \varphi}{2}} \int_{\bar{l}_{2}}^{\bar{l}_{1}} d l\left[\sigma_{\alpha \beta}^{-1} \Delta V(l)-\sigma_{\alpha \beta}^{-1} \Delta V_{1}+v(\varphi)\right]^{-\frac{1}{2}},
$$

where $\Delta V(l)=V(l)-V\left(l_{\pi}\right), \Delta V_{1}=V_{1}-V\left(l_{\pi}\right)$, and $v(\varphi)=\frac{1}{2} \cot ^{2} \varphi \sin \varphi$. $l_{\pi}$ is the equilibrium thickness of the wetting layer on the planar substrate and corresponds to the global minimum of $V(l)$. Eq.(3.4) together with the condition $V\left(\bar{l}_{1}\right)=V\left(\bar{l}_{2}\right)=V_{1}$ allows one to find the pair $\left(\bar{l}_{1}, \bar{l}_{2}\right)$ which characterizes the equilibrium interfacial configuration, see Figs 5 and 6.

\section{IIIb. The free energy decomposition}

The free energy of the system is obtained from the Hamiltonian in Eq.(3.1) evaluated at the equilibrium interfacial configuration $\bar{f}$ :

$$
\mathcal{H}[\bar{f}]=\frac{2 a \sigma_{\alpha \beta}}{\sin \varphi}\left(\sigma_{\alpha \beta}^{-1} \Delta V_{1}-v(\varphi)\right)+
$$




$$
2 \sigma_{\alpha \beta} \sqrt{\frac{2}{\sin \varphi}} \int_{\bar{l}_{2}}^{\bar{l}_{1}} d l\left\{\sqrt{\sigma_{\alpha \beta}^{-1} \Delta V(l)-\sigma_{\alpha \beta}^{-1} \Delta V_{1}+v(\varphi)}-\sqrt{v(\varphi)}\right\} .
$$

Our purpose is to extract the line and the surface contributions to the free energy in Eq.(3.5) in the limit of large $a$ and to discuss their nonanalyticities corresponding to the transitions taking place in the system. This can be - at least partially - achieved via the graphical analyses presented below.

Figures 5 and 6 display the potential difference $\sigma_{\alpha \beta}^{-1} \Delta V(l)$ in the case of the first-order (Figs 5a, 6a) and continuous (Figs 5b, 6b) transitions. Figures 5a,b correspond to $\cos \Theta<\sin \varphi$, i.e. $T<T_{\varphi}$, where $\theta\left(T_{\varphi}\right)=\frac{\pi}{2}-\varphi$. $T_{\varphi}$ is thus identified as the filling temperature for a wedge with the opening angle $2 \varphi$ [22]. Figures 6a,b correspond to $T>T_{\varphi}$. The dotted line represents $\sigma_{\alpha \beta}^{-1} \Delta V_{1}$; its intersections with the plot of $\sigma_{\alpha \beta}^{-1} \Delta V(l)$ determine, together with Eq.(3.4), the equilibrium values of $l_{1}$ and $l_{2}$.

We start with the first-order transition case for $T<T_{\varphi}$ (Fig.5a) and consider the special limit $a \rightarrow \infty$ in which the integral on the r.h.s. of Eq.(3.4) diverges. This divergence occurs either because the integrand diverges or because the upper limit $\bar{l}_{1}$ of integration in Eq.(3.4) diverges. For $T<T_{\varphi}$ only the first possibility can be realized; it happens for $\sigma_{\alpha \beta}^{-1} \Delta V_{1} \nearrow v(\varphi)$. Then the second term of the r.h.s. of Eq.(3.5) remains finite. The first term is a product of two factors; the first factor grows linearly with $a$ and the second one decreases to zero. Thus the first term gives no contribution to the surface free energy; both terms contribute to the line free energy. It is clear from Fig.5b that similar behavior will be observed for the second-order transition as well.

The situation changes for $T>T_{\varphi}$, Figs $6 \mathrm{a}$,b. In the case of the first-order transition (Fig.6a) there is a competition between two solutions corresponding to $\left(\bar{l}_{1}, \bar{l}_{2}\right)$ and $\left(\tilde{l}_{1}, \tilde{l}_{2}\right)$. Note that since both solutions fulfill the constraint given in Eq.(3.4) the values of $\Delta V\left(\bar{l}_{1}\right)=\Delta V\left(\bar{l}_{2}\right)$ and $\Delta V\left(\tilde{l}_{1}\right)=\Delta V\left(\tilde{l}_{2}\right)$ denoted as $\Delta V_{1}$ and $\Delta \tilde{V}_{1}$, respectively are different. The solution $\left(\tilde{l}_{1}, \tilde{l}_{2}\right)$ and the corresponding free energy share the features discussed previously for the case $T \leq T_{\varphi}$ and $a \rightarrow \infty$. In particular, the free energy contains only the line contribution. The solution $\left(\bar{l}_{1}, \bar{l}_{2}\right)$ and the corresponding free energy behave differently; in this case the divergence of the integral on the r.h.s. of Eq.(3.4) is provided by the diverging upper limit of integration. The first term on the r.h.s. of Eq.(3.5) can be rewritten (up to the $(\pi / 2-\varphi)^{2}$ terms) as

$$
2 a \sigma_{\alpha \beta}\left\{\frac{1}{\sin \varphi}\left[\sigma_{\alpha \beta}^{-1} \Delta V_{1}-(1-\cos \theta)\right]+[1-\cos \theta / \sin \varphi]\right\} .
$$

Thus for large $a$ the first term in the above expression ceases to be linear in $a$ while the second term remains negative and proportional to $a$. The integrand in the second term on the r.h.s. of Eq.(3.5) also remains finite in this limit. For $T>T_{\varphi}$ the free energy corresponding to the solution $\left(\bar{l}_{1}, \bar{l}_{2}\right)$ contains an 
additional negative surface contribution. Due to this term the solution $\left(\bar{l}_{1}, \bar{l}_{2}\right)$ corresponds to the actual equilibrium configuration. Since both solutions $\left(\bar{l}_{1}, \bar{l}_{2}\right)$ and $\left(\tilde{l}_{1}, \tilde{l}_{2}\right)$ correspond to the same value of the parameter $a$ one has $\Delta \tilde{V}_{1}>\Delta V_{1}$. For certain range of not too small $a$-values the difference $\mathcal{H}[\tilde{f}]-\mathcal{H}[\bar{f}]$ may change sign depending on the temperature. This marks the existence of the filling temperature $T_{\varphi}(a)$ such that $T_{\varphi}(a)>T_{\varphi}(\infty)=T_{\varphi}$ at which the transition between the two solutions takes place. The difference $T_{\varphi}(a)-T_{\varphi}$ decreases for $a \rightarrow \infty$; this behaviour may be looked upon as the analog of the Kelvin law for the capillary condensation problem. In order to determine the $a$-dependence of $T_{\varphi}(a)-T_{\varphi}$ one has to perform the model dependent numerical analysis which is postponed to future publication. Similar kind of qualitative analysis shows that in the opposite limit, i.e. for $a \rightarrow 0$ one has $T_{\varphi}(a) \rightarrow T_{w}$. For small enough values of parameter $a$ the $\left(\bar{l}_{1}, \bar{l}_{2}\right)$ solution ceases to exist and only the solution $\left(\tilde{l}_{1}, \tilde{l}_{2}\right)$ remains. There is no filling transition in this case.

For a continuous transition, Fig.6b there is only one solution $\left(\bar{l}_{1}, \bar{l}_{2}\right)$. The corresponding free energy contains for large $a$ the negative surface contribution similarly as for the first-order case discussed above.

The above results agree qualitatively with the conclusions of our thermodynamic analysis in Chapter II. At $T=T_{\varphi}$ the surface contributions to the free energy are nonanalytical. They are given by $\frac{2 a}{\sin \varphi} \sigma_{w \alpha}$ for $T \leq T_{\varphi}$ and by $\frac{2 a}{\sin \varphi}\left[\sigma_{w \alpha}+\sigma_{\alpha \beta}(\sin \varphi-\cos \Theta)\right]$ for $T>T_{\varphi}$, see Eq.(2.10). Now we see that also the line contribution which is absent in the thermodynamic analysis turns out to be nonanalytical at $T=T_{\varphi}(a)$. Thus to discuss the line contributions which determine - among others - the interfacial shape one must go beyond the thermodynamic analysis. This will be done in Chapter IV.

\section{IIIc. The wetting transition}

In order to discuss the wetting transition it is necessary to compare the free energy $F[\bar{f}]$ corresponding to the finite solution with the free energy $F_{\infty}$ corresponding to the interface removed infinitely far away from the substrate. This difference is equal

$$
F[\bar{f}]-F_{\infty}=\int_{-a}^{a} d x\left[\frac{1}{2} \sigma_{\alpha \beta}\left(\frac{d f}{d x}\right)^{2}+\frac{\omega(l)}{\sin \varphi}\right],
$$

where $\omega(l)=V(l)-\sigma_{w \beta}-\sigma_{\alpha \beta}$. It can be rewritten with the help of Eq.(3.3) as

$$
F[\bar{f}]-F_{\infty}=\frac{2 a}{\sin \varphi} \omega\left(\bar{l}_{1}\right)+
$$




$$
2 \sigma_{\alpha \beta} \sqrt{\frac{2}{\sin \varphi}} \int_{\bar{l}_{2}}^{\bar{l}_{1}} d l\left\{\sqrt{\sigma_{\alpha \beta}^{-1} V(l)-\sigma_{\alpha \beta}^{-1} V\left(\bar{l}_{1}\right)+v(\varphi)}-\sqrt{v(\varphi)}\right\} .
$$

In the case of the first-order potential $\omega(l)$ both contributions to the free energy difference in Eq.(3.7) are positive at $T_{w}$ which means that the infinite solution is the stable one. At $T_{\varphi}$ both contributions to the free-energy difference in Eq.(3.8) are negative and so the finite solution is the stable one. Thus the wetting transition temperature $T_{w}(a, \varphi)$ is shifted from $T_{w}$ towards $T_{\varphi}$. The magnitude of this shift depends on parameters $a$ and $\varphi$, and the following inequality holds

$$
T_{\varphi}<T_{w}(a, \varphi)<T_{w}
$$

The above result - although derived for the saw-shaped substrate - should also hold for the first-order transitions on other types of periodic and weakly corrugated substrates described by the Hamiltonian in Eq.(3.1).

One should also pay attention to the possibility that the first-order wetting transition on the planar substrate turns into critical filling on the corrugated substrate. This happens for "strongly" corrugated substrate so that the filling temperature $T_{\varphi}(a)$ lies below the spinodal temperature $T_{s}$ at which the potential barrier in $\omega(l)$ dissappears. The presence of this mechanism leading to the change of the order of the transition - which was pointed out in [23] in the context of adsorption on the wedge-shaped substrate - depends on the actual form of the effective potential $\omega(l)$. For certain models of $\omega(l)$ it may be also realized in the case of adsorption on the saw-shaped substrate.

The above reasoning is not valid for the effective potential $V(l)$ exhibiting the continuous transition. The continuous wetting occurs at the same temperature as for the planar case.

When $T \nearrow T_{w}$ then $\bar{l}_{2}, \bar{l}_{1}, l_{\pi}$ increase indefinitely and $\omega\left(l_{\pi}\right) \rightarrow 0$. In consequence one can neglect the potential terms in Eq.(3.4) and derive the relation

$$
a \approx\left(\bar{l}_{1}-\bar{l}_{2}\right) \tan \varphi
$$

This relation becomes exact at $T=T_{w}$. At this temperature the interface is flat and situated infinitely far away from the substrate.

\section{Analytic results for continuous filling}

In this chapter we discuss the case when the effective potential $V(l)$ corresponds to the critical wetting on the planar substrate

$$
V(l)=\sigma_{w \beta}+\sigma_{\alpha \beta}+W_{0} \tau \exp (-l / \xi)+U_{0} \exp (-2 l / \xi)
$$

where the parameter $\tau \leq 0$ measures the distance from the planar substrate wetting point $\tau_{w}=0$, and the constants $W_{0}, U_{0}$ are positive. The solution of 
Eq.(3.2) has the following form

$$
\bar{l}(x)=l_{0}+\xi \ln [1+A \exp (\lambda x)+B \exp (-\lambda x)],
$$

where the constants $l_{0}, \lambda, A, B$ are determined by requiring that $\bar{l}(x)$ fulfills Eq.(3.2) and the boundary conditions $\bar{l}^{\prime}\left(0^{+}\right)=\bar{l}^{\prime}\left(a^{-}\right)=-\cot \varphi$. The solution $\bar{l}(x)$ can be conveniently rewritten using the following dimensionless variables $y=\lambda \xi / \cot \varphi, t=\tau / \tau_{\varphi}, \tau_{\varphi}=-\sqrt{2 U_{0} \sigma_{\alpha \beta} W_{0}^{-2} \sin ^{-1} \varphi} \cos \varphi, \bar{a}=a \cot \varphi / \xi$, $\chi=x \cot \varphi / \xi . \quad \bar{a}$ measures the dimensionless depth of the depression at its center and $\tau_{\varphi}<0$ denotes a characteristic temperature for the adsorption on the saw-like substrate. For large opening angles considered in this paper $\tau_{\varphi}$ coincides with the wedge filling temperature up to the terms $0(\pi / 2-\varphi)^{2}$. $t$ measures the dimensionless temperature: $t>1$ corresponds to temperature below the characteristic temperature $\tau_{\varphi}$. Then

$$
\begin{gathered}
\left(y^{2}-1\right)\left(y^{2}-t^{2}\right)=t^{2} \cosh ^{-2}(y \bar{a} / 2), \\
\bar{l}(x)=l_{0}+\xi \ln \left[1-\frac{y \cosh (y \chi)-\sinh (y \chi)}{\left(y^{2}-1\right) \sinh (y \bar{a})}+\right. \\
\left.+\frac{y \cosh (y(\chi-\bar{a}))-\sinh (y(\chi-\bar{a}))}{\left(y^{2}-1\right) \sinh (y \bar{a})}\right],
\end{gathered}
$$

and

$$
l_{0}=l_{\pi}-2 \xi \ln \left(\frac{y}{t}\right),
$$

where $l_{\pi}=-\xi \ln \left(t \cos \varphi \sqrt{\frac{\sigma_{\alpha \beta}}{2 U_{0} \sin \varphi}}\right)$. The solutions of Eq.(4.3) - which serve as the input to Eq.(4.4) - are parametrized by the temperature $t$ and the geometry of the substrate, i.e. $a$ and $\varphi$. The shape of the emerging interface is shown on Fig. 7 for different temperatures. Fig. 8 shows $\bar{l}_{1}$ and $\bar{l}_{2}$ as functions of the reduced temperature; especially pronounced is the sharp increase of $\bar{l}_{1}$ upon crossing the wedge filling temperature $t=1$. This means that the trace of the filling transition chracteristic for the adsorption in the wedge is also present for the saw-shaped substrate, although not in such a singular form as for the wedge. Also the width of the adsorbed layer at $x=a$, i.e. $\bar{l}_{2}$ does not exhibit any nonanalytic behavior near $t=1$; see Fig.8. A straightforward calculation shows that the free energy $F[\bar{f}]$ evaluated at $t=1$ tends to a constant for $a \rightarrow \infty$; under the same conditions the first derivative of $F[\bar{f}]$ with respect to the temperature grows linearly with $a$. This behaviour of the free energy in the limit $a \rightarrow \infty$ is compatible with our previous mean-field results obtained for the wedge [22]. On the other hand, upon approaching the planar substrate wetting temperature $t=0$ both $\bar{l}_{1}$ and $\bar{l}_{2}$ grow indefinitly which reflects the wetting transition taking place on this periodically corrugated substrate. 
An interesting insight into the structure of the interfacial profile $\bar{f}(x)$ can be obtained by analyzing the scaling properties of $\bar{l}(x)$ in the limit of large $\bar{a}$ for different temperature regimes.

It follows from Eq.(4.4) that the film thicknesses $\bar{l}_{1}$ and $\bar{l}_{2}$ are given by

$$
\bar{l}_{1}=l_{0}+\xi \ln \left[\frac{y\left[y+\tanh \left(\frac{y \bar{a}}{2}\right)\right]}{\left(y^{2}-1\right)}\right]
$$

and

$$
\bar{l}_{2}=l_{0}+\xi \ln \left[\frac{y\left[y-\tanh \left(\frac{y \bar{a}}{2}\right)\right]}{\left(y^{2}-1\right)}\right],
$$

where the value of parameter $y(t, \bar{a})$ is obtained by solving Eq.(4.3). After substituting the relevant solutions into Eq.(4.4) one obtains

$$
\bar{l}_{1} \approx l_{\pi}+\left\{\begin{array}{cl}
\xi \ln \left(\frac{t}{t-1}\right) & \text { for } t>1 \\
\bar{a} \xi / 2 & \text { for } t=1 \\
\bar{a} \xi+\xi \ln \left(\frac{1-t^{2}}{2}\right) & \text { for } t<1
\end{array}\right.
$$

where the expressions in Eq.(4.8) hold for $t \bar{a} \exp (-t \bar{a}) \ll\left(t^{2}-1\right),(t>1)$, and $t \bar{a} \exp (-\bar{a}) \ll\left(1-t^{2}\right),(t<1)$, respectively. Thus for $\bar{a} \rightarrow \infty$ the scaling behavior of $\bar{l}_{1}$ depends on the chosen temperature regime. For $t>1$ the value of $\bar{l}_{1}$ approaches the width of the adsorbed layer in the wedge. For $t \leq 1$ the value of $\bar{l}_{1}$ grows linearly with $\bar{a}$; the slope of this linear increase is $\frac{1}{2}$ for $t=1$, and 1 for $t<1$. This change of slope is visible in Fig.9 which illustrates the scaling properties of $\bar{l}_{1}$ for different temperatures.

The scaling properties of $\bar{l}_{2}$ are different from those of $\bar{l}_{1}$. For $t \rightarrow 0 \quad \bar{l}_{2} \rightarrow l_{\pi}$ and $l_{\pi}$ itself increases logarithmically. Thus for $t \rightarrow 0$ both $\bar{l}_{1}$ and $\bar{l}_{2}$ approach $l_{\pi} ; \bar{l}_{1}-\bar{l}_{2} \rightarrow \xi \bar{a}$, i.e. $\bar{f}(0)-\bar{f}(a) \rightarrow 0$. The interface becomes flat and the system undergoes the continuous wetting transition.

\section{The summary}

Our main conclusion is that the complete scenario of adsorption on a corrugated substrate contains both the filling and the wetting transition. The filling transition corresponds to either partial or complete filling of the substrate's depressions. Then follows the wetting transition at which the whole substrate becomes covered by a macroscopically thick layer of the adsorbed phase. The detailed scenario of the filling transition depends on the substrate's convexity properties. Thermodynamic considerations show that for periodic substrates which are piecewise convex, i.e. convex except for isolated points at which the substrate shape is nonanalytic, one has continuous growth of the adsorbed phase 
which - with increasing temperature - fills the substrate's depressions starting from its bottom and terminating at its top. The substrate's top position, i.e $z=b(a)$ is reached by the $\alpha-\beta$ interface at the wetting temperature $T_{w}$. On the other hand, for periodic substrates which are piecewise concave one has discontinuous growth. The $\alpha-\beta$ interface jumps to the position at the top of the substrate at the temperature $T_{f}<T_{w}$. For periodic substrate shapes like $b(x)=a[1-\cos (\pi x / a)]$ which within each section contain both concave and convex parts the filling scenario corresponds to the jump of the interface to the position where it makes contact with the convex part of the substrate. Upon further increase of the temperature this jump is then followed by a continuous growth of the interfacial position until the whole depression becomes filled.

An interesting situation appears when - within each section of the substrate - there are more than one inflection point. This corresponds to varying convexity properties of the substrate which are then reflected in the course of the filling transition. It consists of jumps followed by continuous growth after which another jump comes etc. This highly nonuniversal scenario requires a detailed analyses which is postponed to future publication.

As far as the wetting transition is concerned we argue that its order is the same as in the case of the corresponding planar substrate. The mesoscopic part of our analysis is focused on adsorption on a saw-like substrate which is the borderline case between piecewise convex and piecewise concave substrate shapes. When the substrate is chosen in such a way that in the planar case it corresponds to the critical wetting then the filling transition can be discussed to much extend analytically. Especially we analyze the behavior of the interfacial shape in the vicinity of the wedge filling temperature $T_{\varphi}$ and we point at a very sharp but smooth changes of the interfacial shape near this temperature. We also derive the scaling behavior of $\bar{l}_{1}$ with respect to increasing depression size for temperatures below and above this special temperature.

When the substrate is chosen such that in the planar case it corresponds to the first-order wetting then we show that the filling transition on the periodically corrugated substrate is also first-order but shifted to a higher temperature $T_{\varphi}(a): T_{\varphi} \leq T_{\varphi}(a)$. This scenario holds unless it is preempted by a continuous adsorption which may take place on the saw-shaped substrate when the filling transition temperature $T_{\varphi}(a)$ is shifted below the spinodal temperature $T_{s}$. We also observe that it is actually the line contribution to the free energy that becomes nonanalytic at $T_{\varphi}(a)$. On the contrary, the shift of the saw-shaped substrate wetting temperature $T_{w}(a, \varphi)$ with respect to the wedge wetting temperature $T_{w}$ is such that $T_{w}(a, \varphi)<T_{w}$. Thus one has $T_{\varphi}<T_{\varphi}(a)<T_{w}(a, \varphi)<T_{w}$.

\section{ACKNOWLEDGEMENTS}

The authors gratfully acknowledge the discusssions with Siegfried Dietrich 
and Andrew Parry, and the support by the Foundation for German-Polish Collaboration under Grant. No. 3269/97/LN. 


\section{Referrences}

[1] P.G. de Gennes, Rev.Mod.Phys. 57, 827 (1985).

[2] S. Dietrich, in Phase Transitions and Critical Phenomena, edited by C. Domb and J.L. Lebowitz (Academic, London, 1988), Vol.12, p. 1.

[3] M. Schick, in Liquids at Interfaces, Proceedings of the Les Houches Summer School in Theoretical Physics, Session XLVIII, edited by J. Chavrolin, J. F. Joanny, and J. Zinn-Justin (North-Holland, Amsterdam, 1990), p. 415.

[4] D. Andelman, J.F. Joanny, and M. O. Robbins, Europhys. Lett. 7, 731 (1988); M. O. Robbins, D. Andelman, J. F. Joanny, Phys. Rev. A 43, 4344 (1991); J. L. Harden and D. Andelman, Langmuir 8, 2547 (1992).

[5] P. Pfeifer, Y.J. Wu, M.W. Cole, and J. Krim, Phys. Rev. Lett. 62, 1997 (1989); P. Pfeifer, M.W. Cole, and J. Krim, Phys. Rev. Lett. 65, 663 (1990).

[6] M. Kardar and J.O. Indekeu, Phys. Rev. Lett. 65, 662 (1990); Europhys. Lett. 12, 161 (1990); H. Li and M. Kardar, Phys. Rev. B 42, 6546 (1990); J. Krim and J.O. Indekeu, Phys. Rev. E 48, 1576 (1993).

[7] E. Cheng, M.W. Cole, and A.L. Stella, Europhys. Lett. 8, 537 (1989); E. Cheng, M.W. Cole, and P. Pfeifer, Phys. Rev. B 39, 12962 (1989).

[8] G. Giugliarelli and A.L. Stella, Phys. Scripta T 35, 34 (1991); Phys. Rev. E 53, 5035 (1996); Physica A 239, 467 (1997); G. Sartoni, A.L. Stella, G. Giugliarelli, and M.R. D’Orsogna, Europhys. Lett. 39, 633 (1997).

[9] M. Napiórkowski, W. Koch, and S. Dietrich, Phys. Rev. A 45, 5760 (1992).

[10] G. Palasantzas, Phys. Rev. B 48, 14472 (1993); Phys. Rev. B 51, 14612 (1995).

[11] J.Z. Tang and J.G. Harris, J. Chem. Phys. 103, 8201 (1995).

[12] A. Marmur, Langmuir 12, 5704 (1996).

[13] C. Borgs, J. De Coninck, R. Kotecký, and M. Zinque, Phys. Rev. Lett. 74, 2293 (1995); K. Topolski, D. Urban, S. Brandon, and J. De Coninck, Phys. Rev. E 56, 3353 (1997).

[14] R. Netz and D. Andelman, Phys. Rev. E 55, 687 (1997).

[15] A.O. Parry, P.S. Swain, and J.A. Fox, J. Phys.: Condens. Matter 8, L659 (1996); P.S. Swain and A.O. Parry, J. Phys. A: Math. Gen. 30, 4597 (1997). 
[16] K. Rejmer and M. Napiórkowski, Z. Phys. B 102, 101 (1997).

[17] P.S. Swain and A.O. Parry, Eur. Phys. J. B4, 459 (1998).

[18] S. Dietrich, in Proceedings of the NATO-ASI New Approaches to Old and New Problems in Liquid State Theory - Inhomogeneities and Phase Separation in Simple, Complex and Quantum Fluids, Vol.C529 of NATO Advanced Study Institute, Messina, Italy, 1998, edited by C. Caccamo (Kluwer, Dordrecht,1999), p.197.

[19] C. Rascón, A.O. Parry, and A. Sartori, preprint cond-mat/9902070

[20] Y. Pomeau, J. Coll. Interf. Sci. 113, 5 (1985).

[21] E.H. Hauge, Phys. Rev. A 46, 4944 (1992).

[22] K.Rejmer, S.Dietrich, and N.Napiórkowski, Phys. Rev. E 60, 4027 (1999).

[23] A.O. Parry, C. Rascón, and A.J. Wood, preprint (1999).

[24] R.N. Wenzel, J. Phys. Colloid Chem. 53, 11466 (1949); Ind. Eng. Chem. 28, 988 (1936).

[25] P.S. Swain, R. Lipowsky, Langmuir 14, 6772 (1998).

[26] P. Lenz and R. Lipowsky, Phys. Rev. Lett. 80, 1920 (1998). 


\section{Figure captions}

Fig.1. Schematic configurations of the $\alpha$ - $\beta$ interface at the depression extending for $x \in[-a, a] . x_{1}$ is the abscissa's value at which the interface makes contact with the substrate; $\psi$ denotes the actual contact angle. (a) the partial filling of the depression, (b) the interface located above the substrate.

Fig.2. Plots of the excess free energy $\Delta F\left(x_{1}\right)$ corresponding to the substrate's shape $b(x)=a(1-\cos (\pi x / a))$ for different values of the temperature. The curve in the middle corresponds to the temperature $T=T_{f}$ at which the first-order transition from the so-called empty depression, i.e. $\bar{x}_{1}=0$ to a partially filled depression with $\bar{x}_{1}=\bar{x}_{1 f}<a$ takes place.

Fig.3. Schematic plots of the excess free energy $\Delta F\left(x_{1}\right)$ corresponding to piecewise concave substrate for different temperatures. An example of such $\Delta F\left(x_{1}\right)$ is given in Eq.(2.7). The curve in the middle corresponds to the temperature at which the whole depression becomes filled at the first-order filling transition.

Fig.4. Schematic plots of the excess free energy $\Delta F\left(x_{1}\right)$ corresponding to piecewise convex substrate for different temperatures. An example of such $\Delta F\left(x_{1}\right)$ is given in Eq.(2.8). The filling of the depression proceeds continuously and terminates at the planar substrate wetting temperature $T_{w}$ at which the whole depression becomes filled. This corresponds to the lower curve which minimum is located at $x_{1}=a$.

Fig.5. The shape of the effective interface potential $\sigma_{\alpha \beta}^{-1} \Delta V(l)$ for $T<T_{\varphi}$ in the case of the first-order wetting transition on the corresponding planar substrate (a), and in the case of the continuous wetting transition on the corresponding planar substrate (b). The dashed lines denote the function $v(\varphi)$, the dotted lines $-\sigma_{\alpha \beta}^{-1} \Delta V_{1}$. $\bar{l}_{1}$ and $\bar{l}_{2}$ are the equilibrium values of the width of the absorbed layer at $x=0$ and $x=a$, respectively. $1-\cos \theta$ is the limiting value of $\sigma_{\alpha \beta}^{-1} \Delta V(l)$ for $l \rightarrow \infty$ and is denoted by thin dashed lines. $\sigma_{\alpha \beta}^{-1} \Delta V\left(l_{\pi}\right)=0$; the value of $l_{\pi}$ which gives the equilibrium width of the wetting layer on the corresponding planar substrate is not marked on this figure. The dotted line approaches the dashed line for $T \nearrow T_{\varphi}$.

Fig.6. The shape of the effective interface potential $\sigma_{\alpha \beta}^{-1} \Delta V(l)$ for $T>T_{\varphi}$ in the case of the first-order wetting transition on the corresponding planar substrate (a), and in the case of the continuous wetting transition on the corresponding planar substrate (b). The notation is the same as in Fig.5. In the case of the first-order wetting there exist two solutions described in the text as $\left(\tilde{l}_{1}, \tilde{l}_{2}\right)$ and $\left(\bar{l}_{1}, \bar{l}_{2}\right)$. For clarity reasons only $\tilde{l}_{1}$ is marked on Fig.6a; $\tilde{l}_{2}$ practically coincides with $\bar{l}_{2}$. For the same reasons we have not plotted separately horizontal dotted 
lines corresponding to $\Delta \tilde{V}_{1}$ and $\Delta V_{1}$ although they differ in values. For $T \searrow T_{\varphi}$ the dotted line approaches the asymptote $1-\cos \theta$ while $\bar{l}_{1} \rightarrow \infty$.

Fig.7. The shapes of the $\alpha-\beta$ interface for different dimensionless temperatures approaching the temperature $t=1$ which corresponds to the critical filling transition on the saw-shaped substrate. The three curves, in increasing order, correspond to the temperatures $t=1.2, t=1$, and $t=0.9$, respectively.

Fig.8. The widths of the adsorbed layer $\bar{l}_{1}$ and $\bar{l}_{2}$ as functions of the dimensionless temperature $t$. For $t \searrow 0$ both $\bar{l}_{1}$ and $\bar{l}_{2}$ tend to $\infty$ with their difference kept constant and equal to $a$; on this figure $\bar{a}=10$.

Fig.9. The scaling behaviour of $\bar{l}_{1}=\bar{f}(0)$ as function of $a$ for different temperatures in the vicinity of $t=1$. For $t=1 \bar{l}_{1} \sim \bar{a} / 2$, for $t<1 \bar{l}_{1} \sim \bar{a}$. For $t>1 \bar{l}_{1}$ approaches the value corresponding to the adsorption in the wedge. 


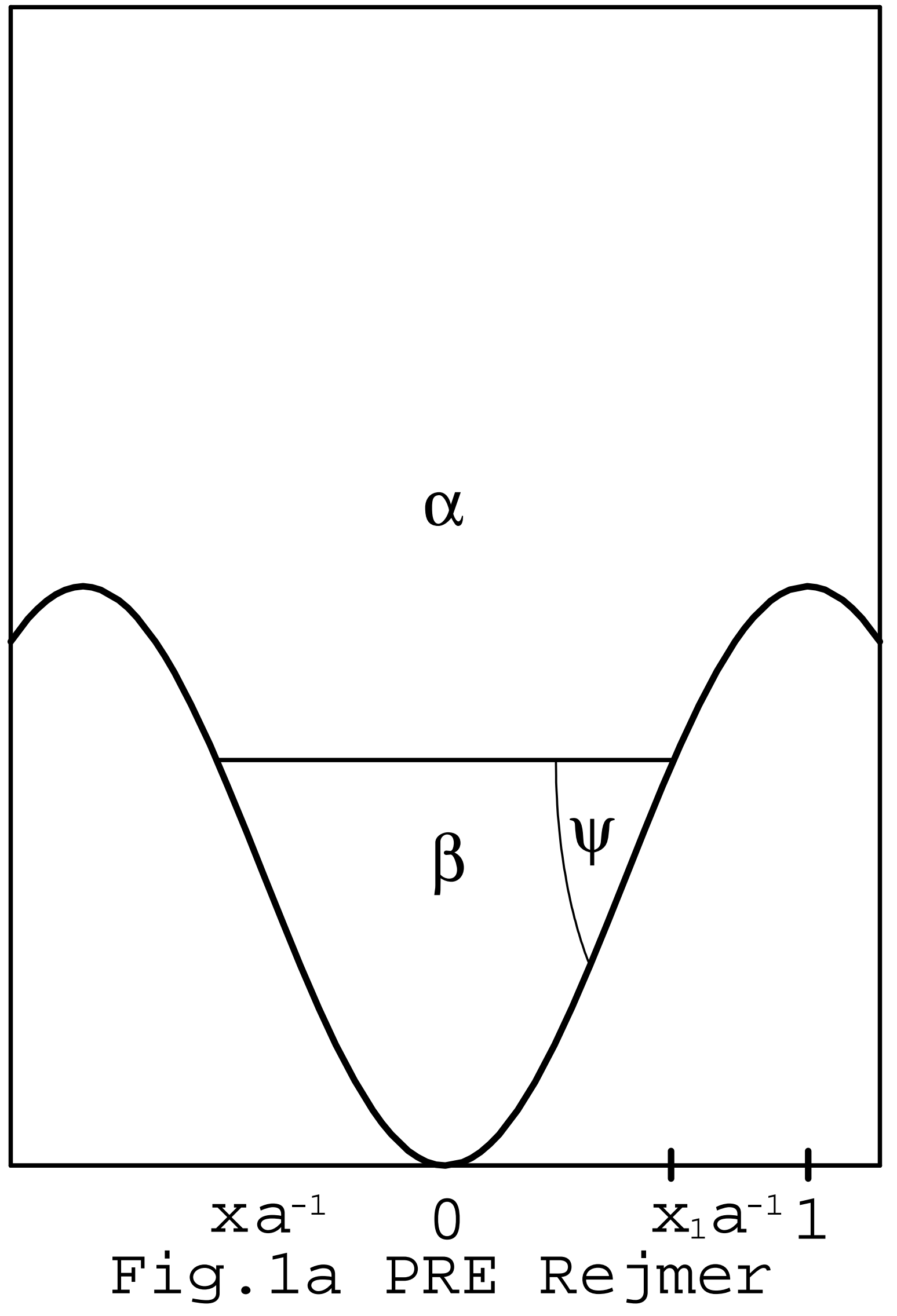




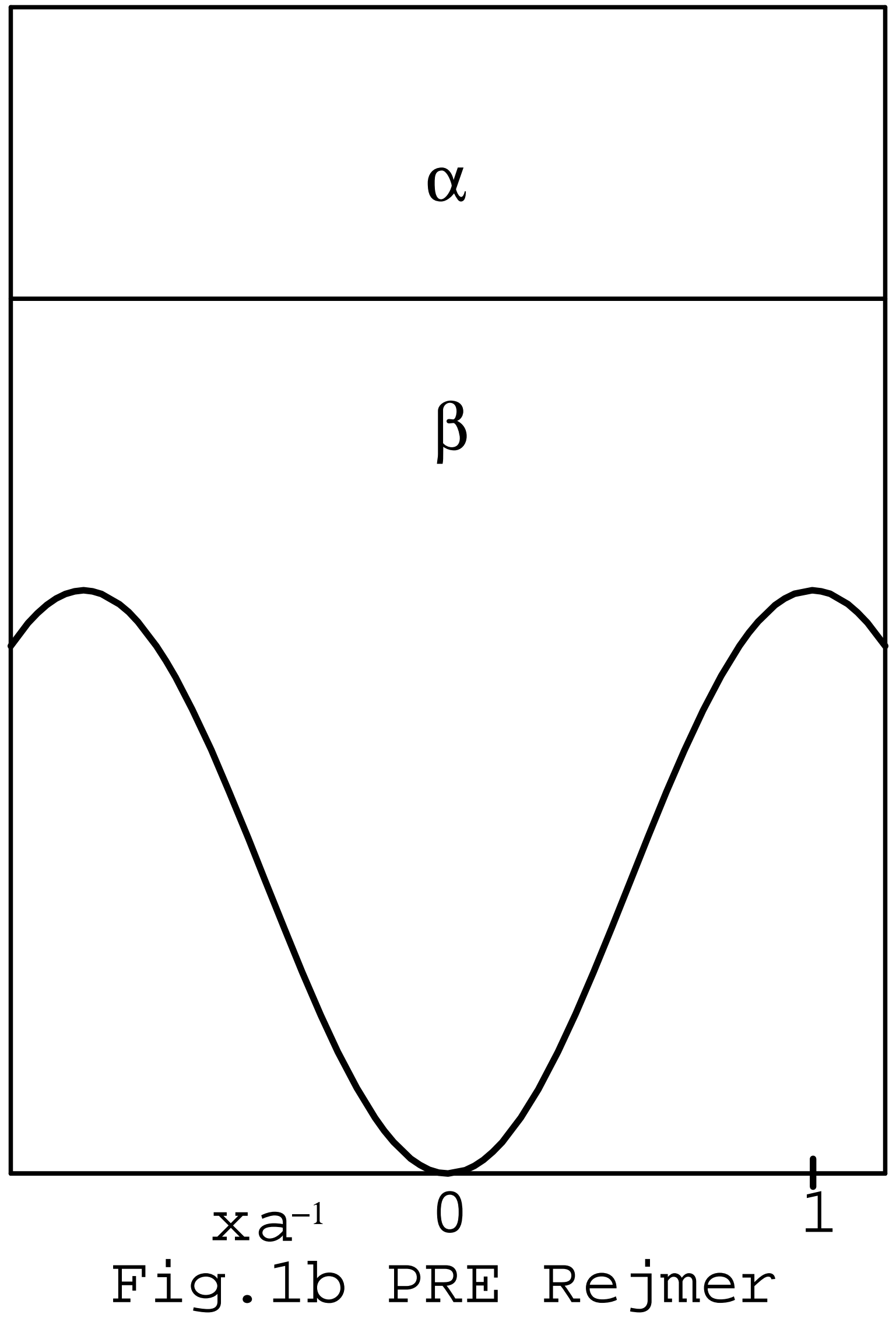




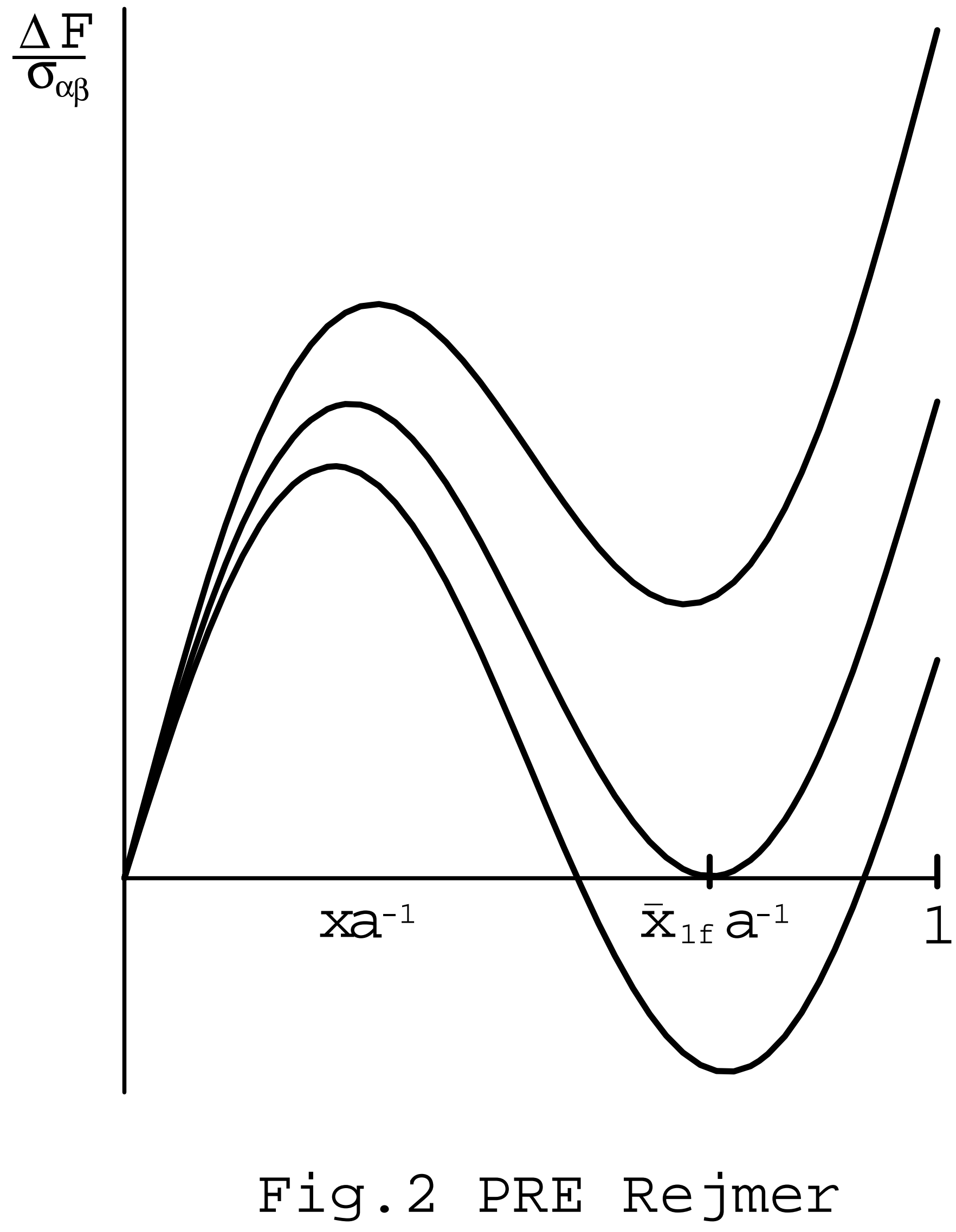




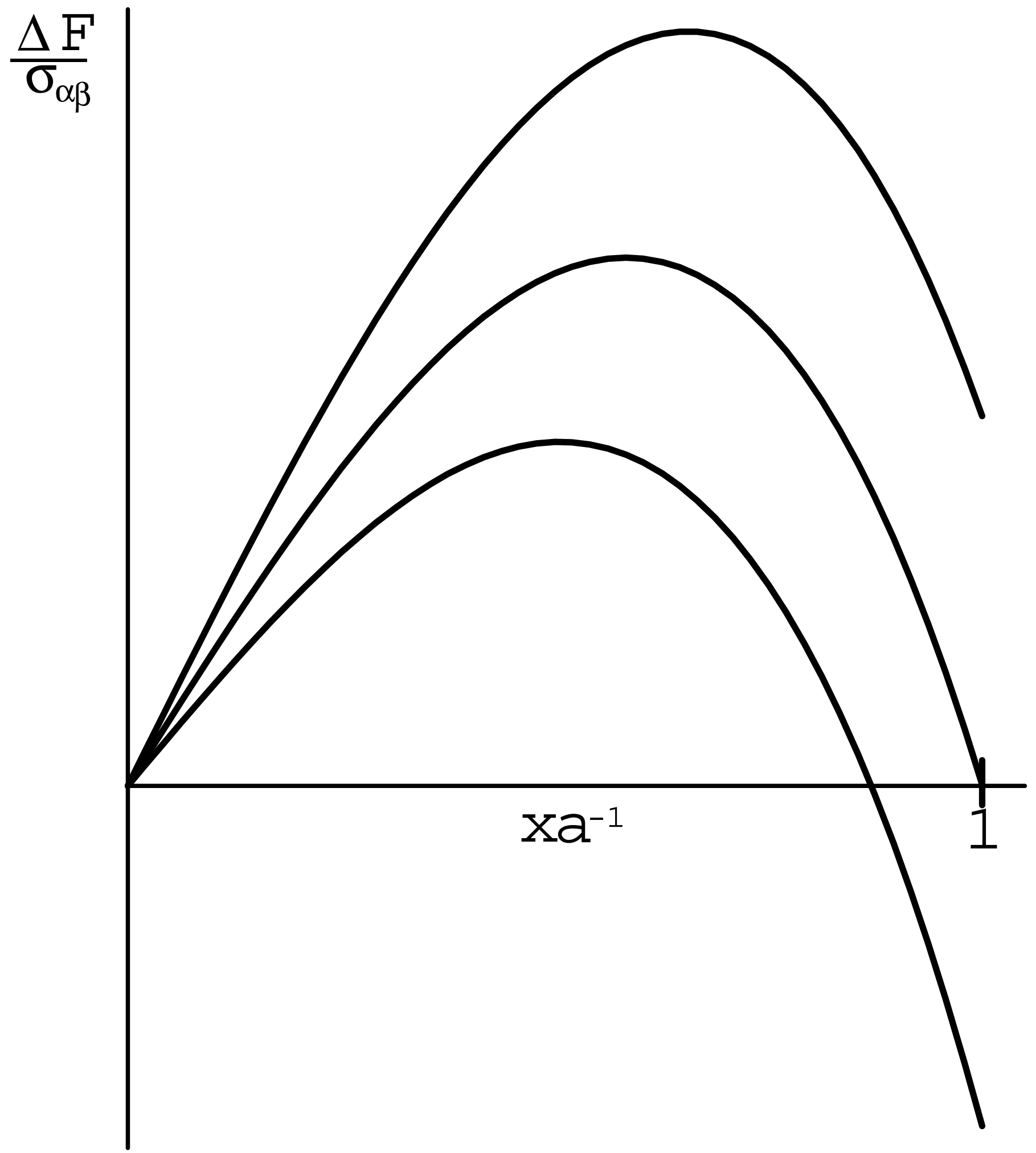

Fig.3 PRE Rejmer 


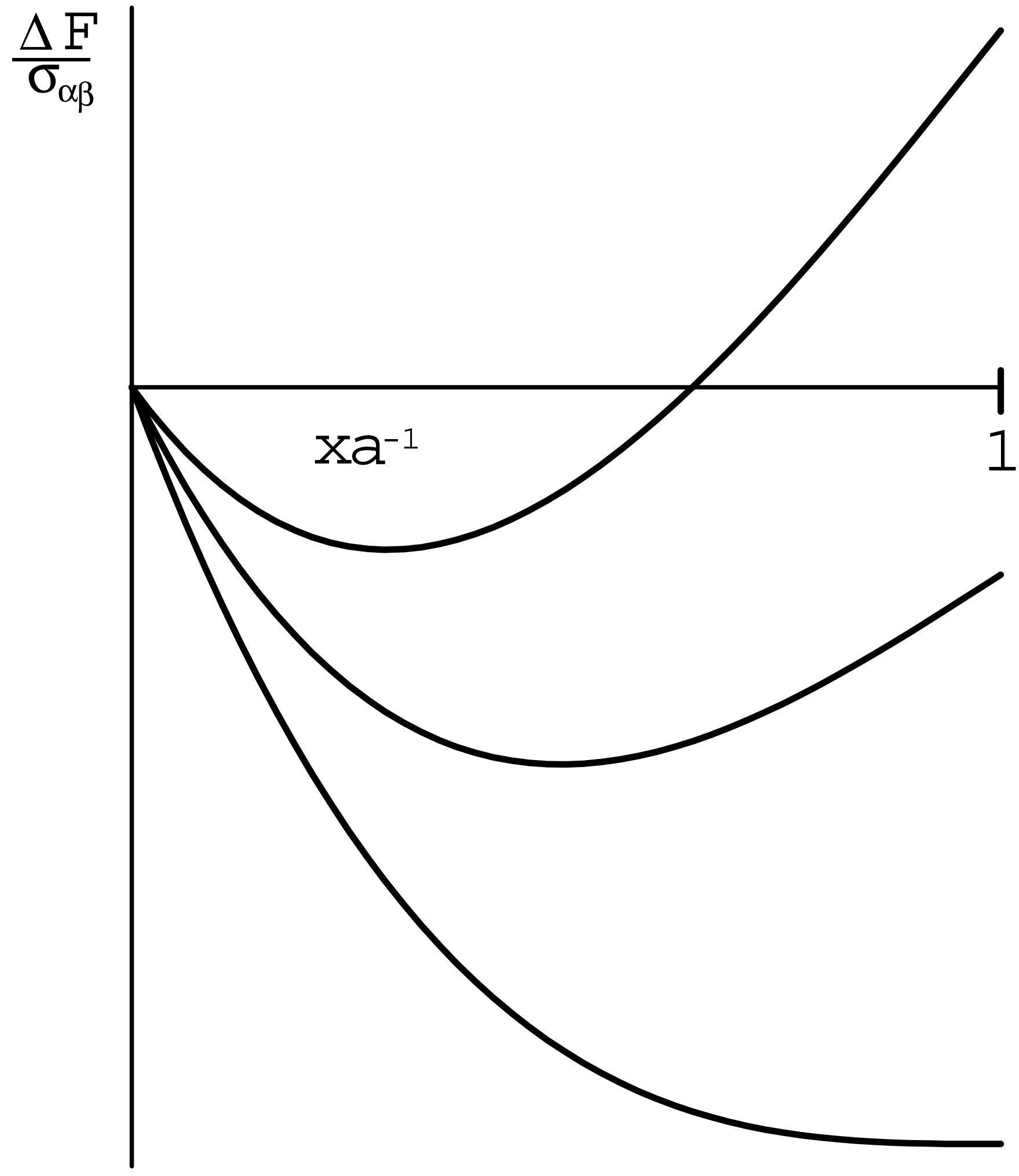

Fig.4 PRE Rejmer 


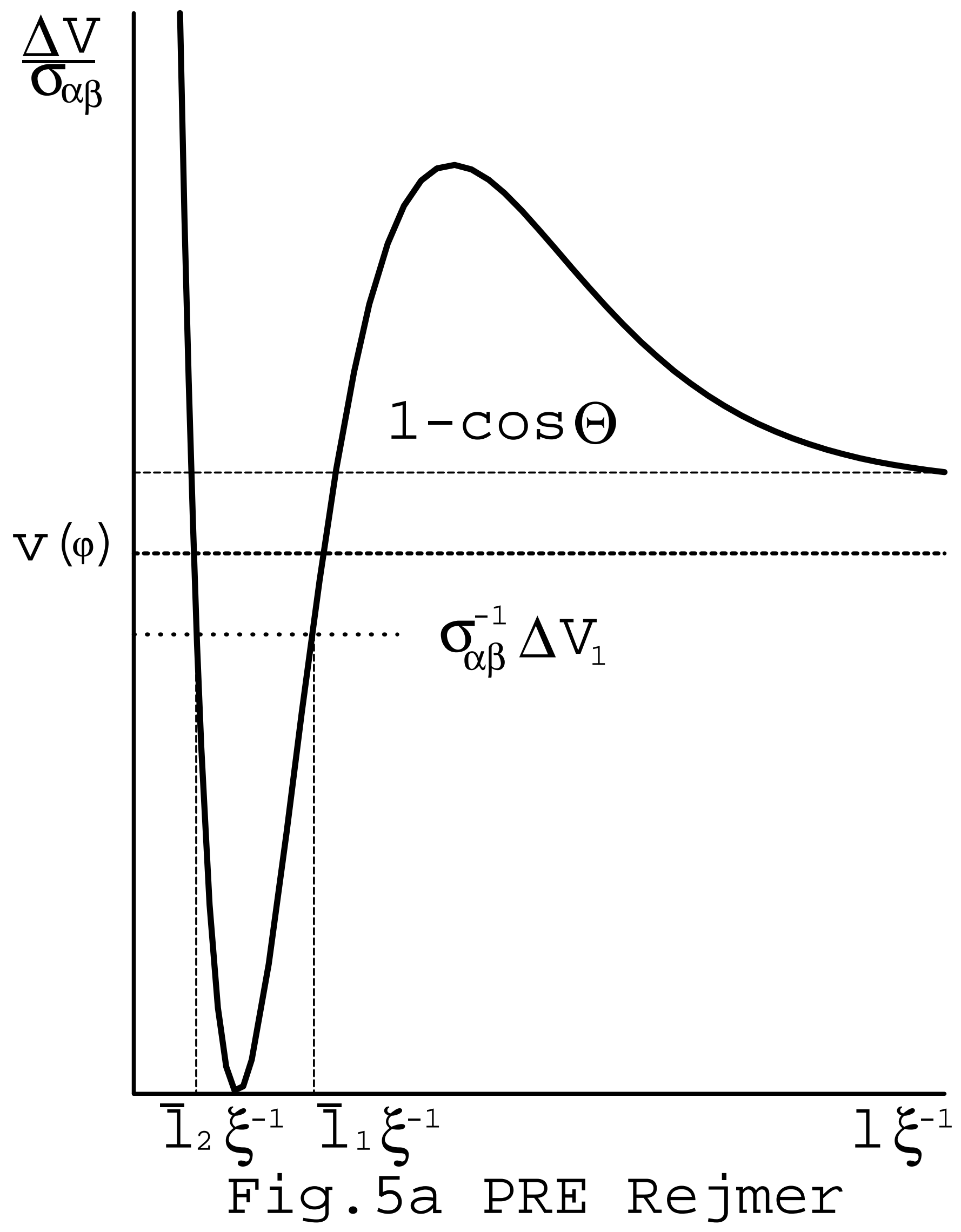




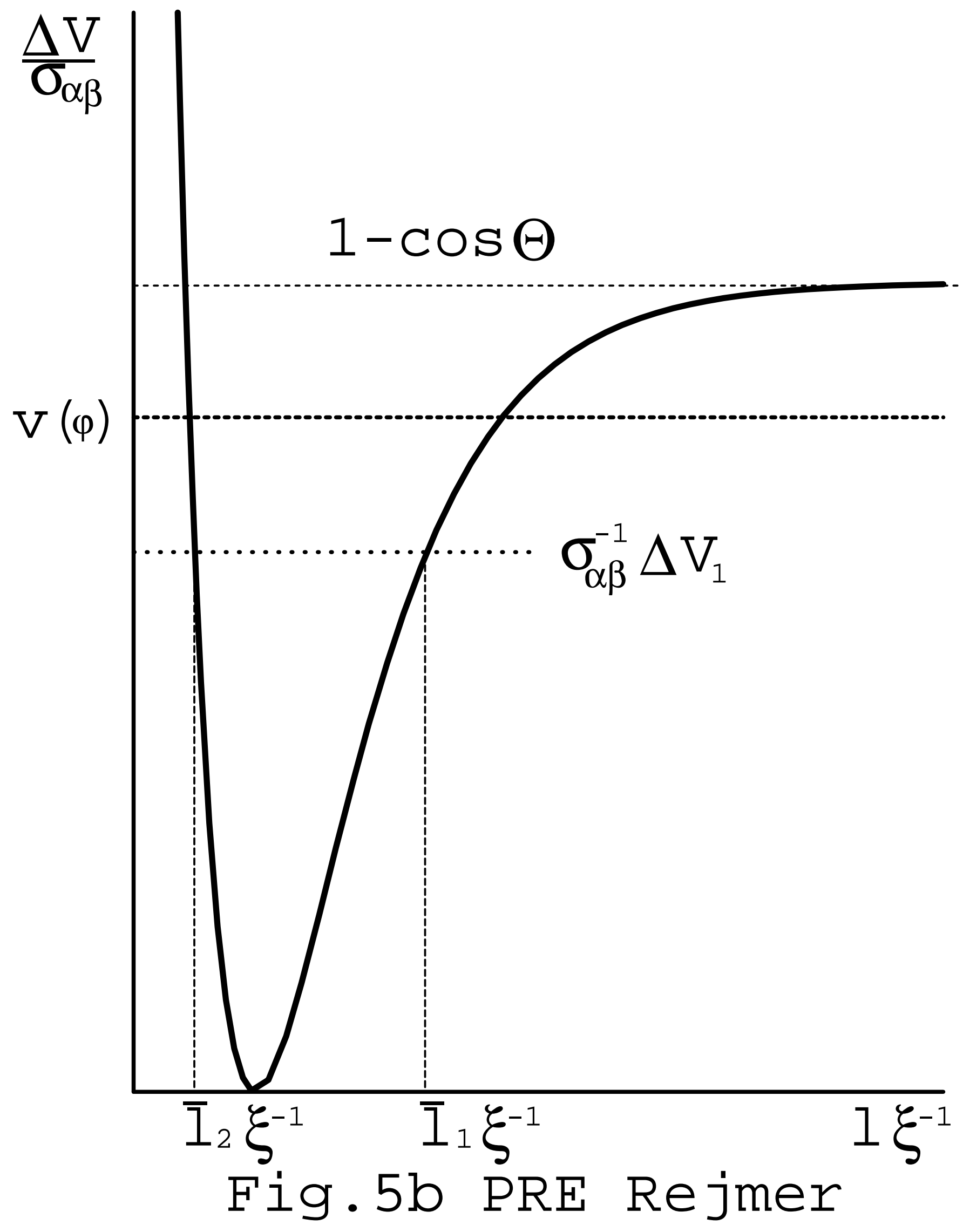




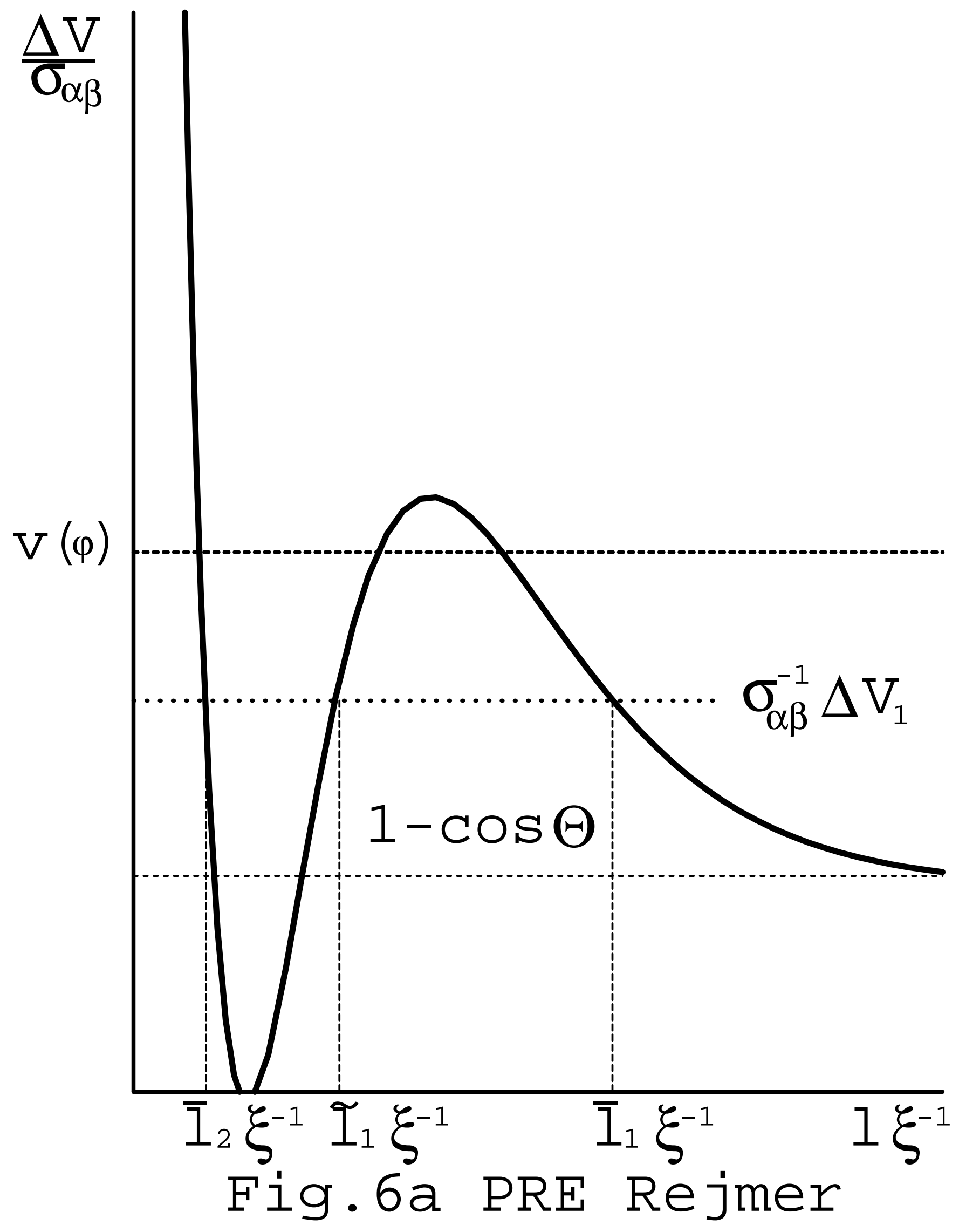




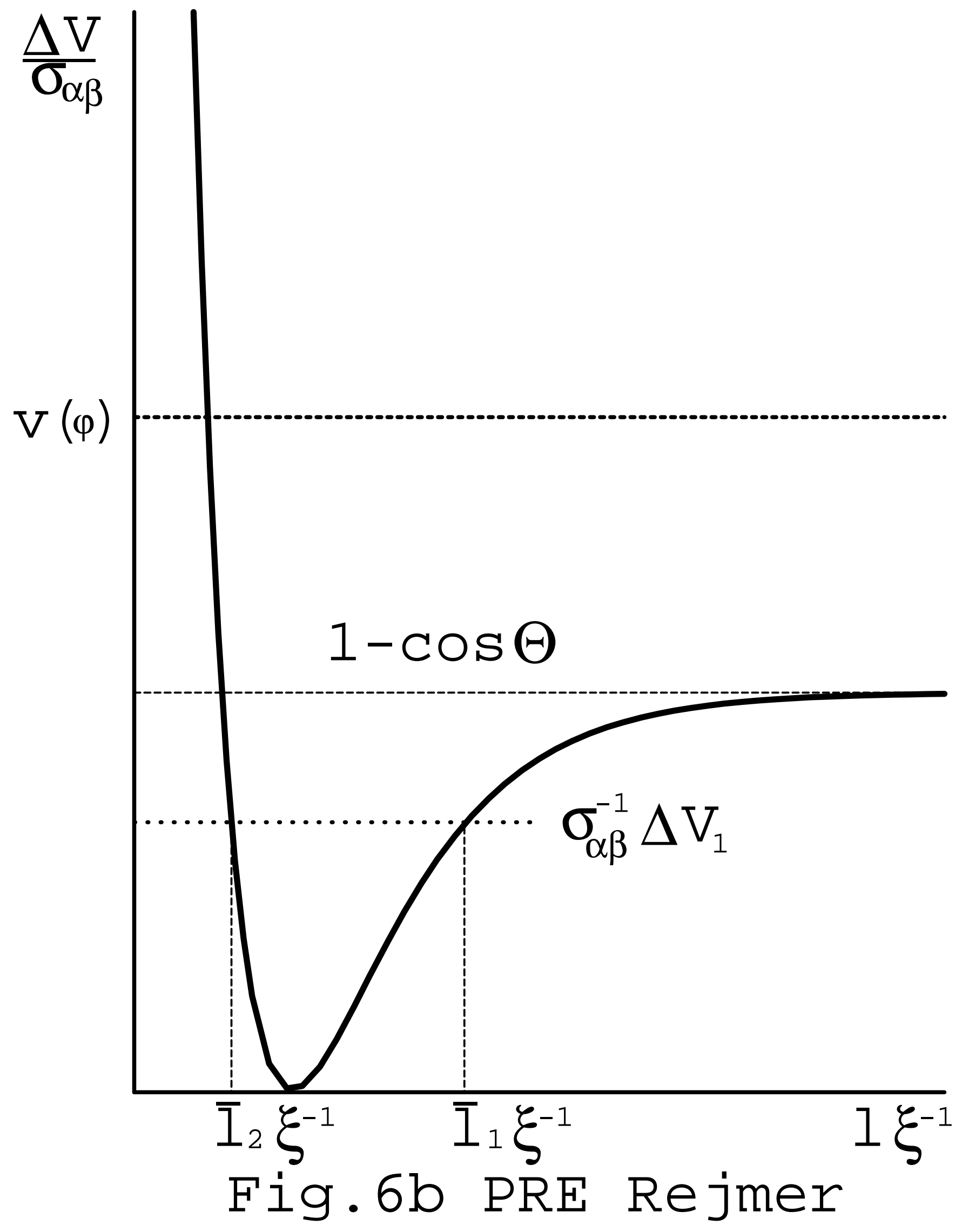




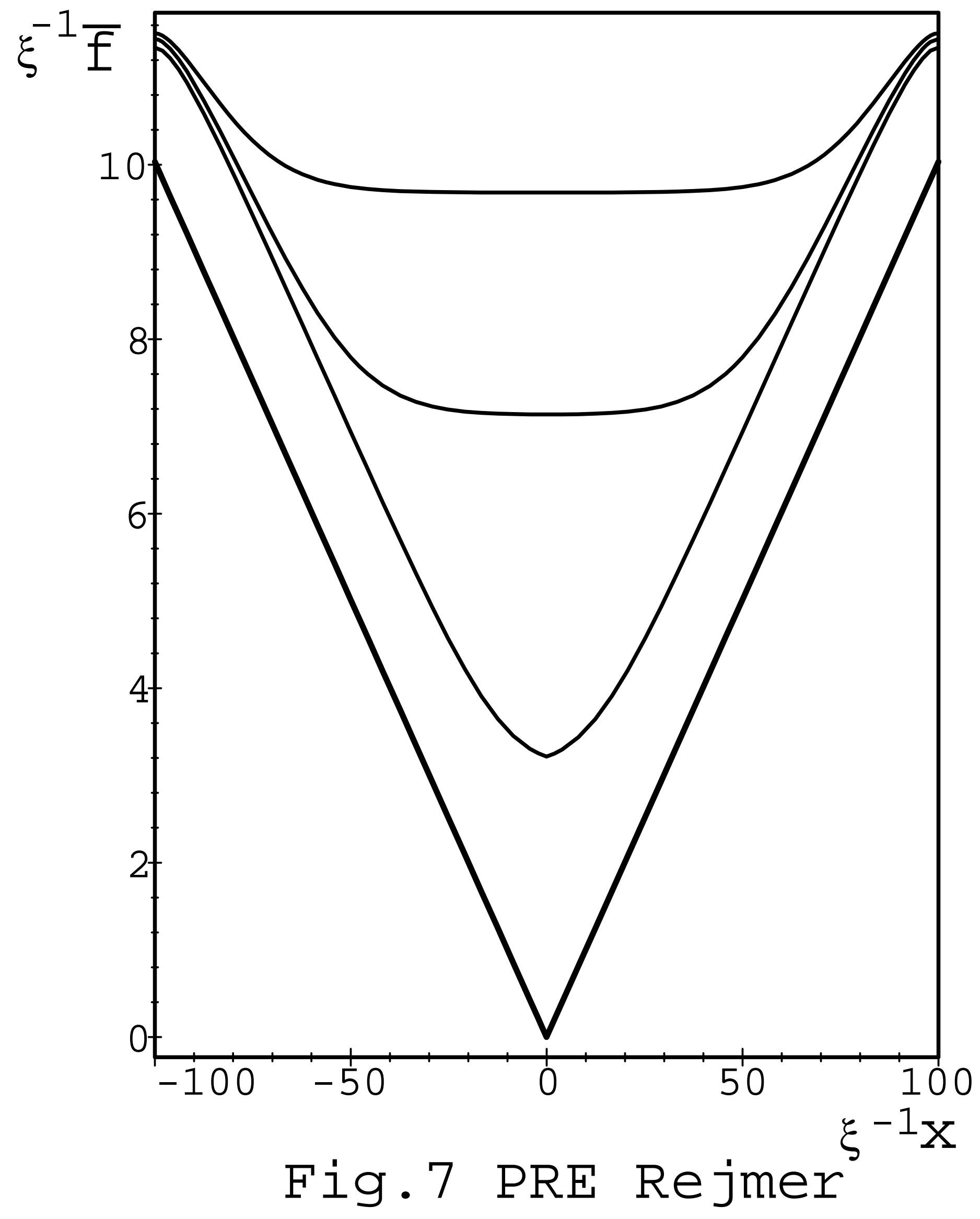




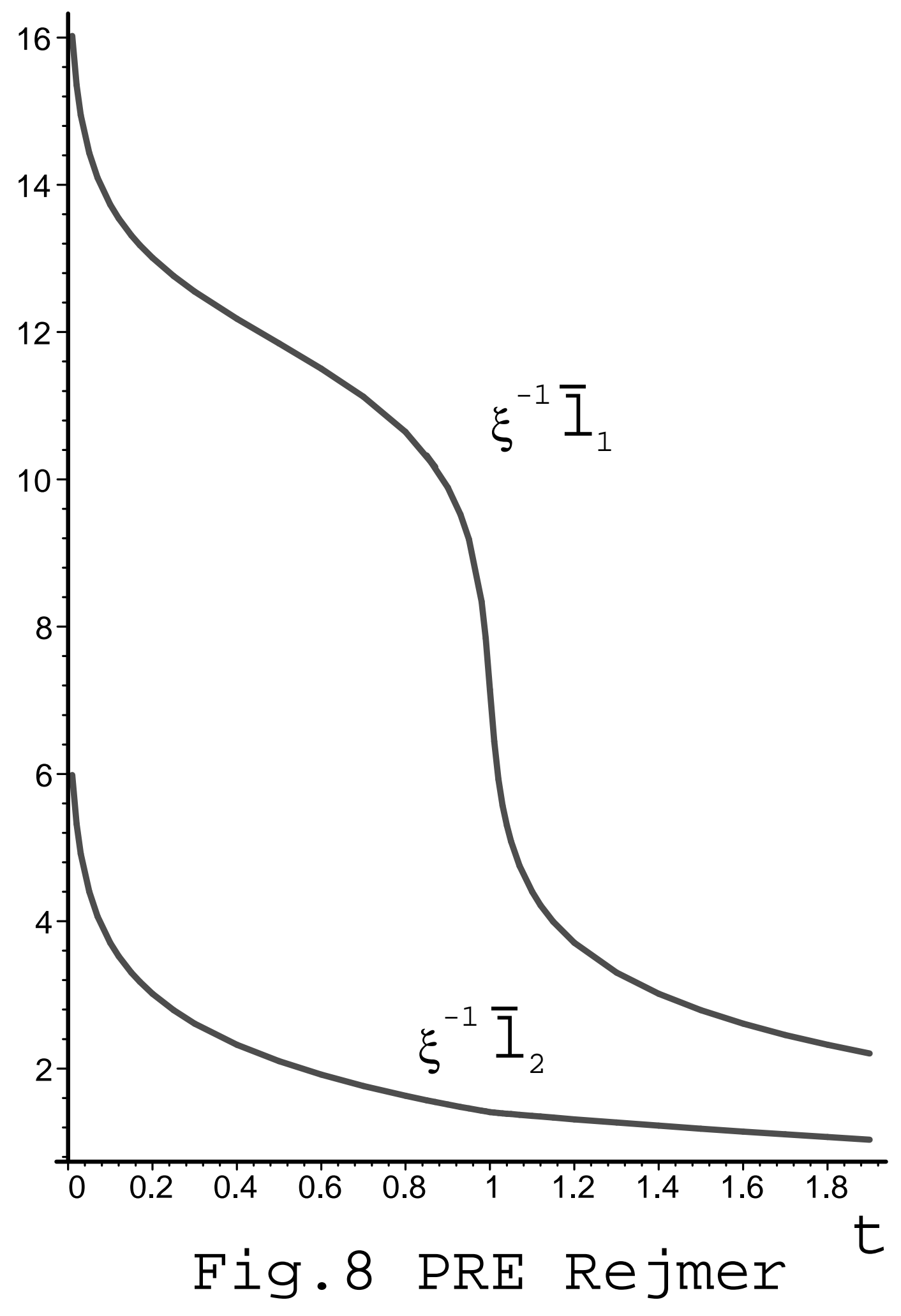




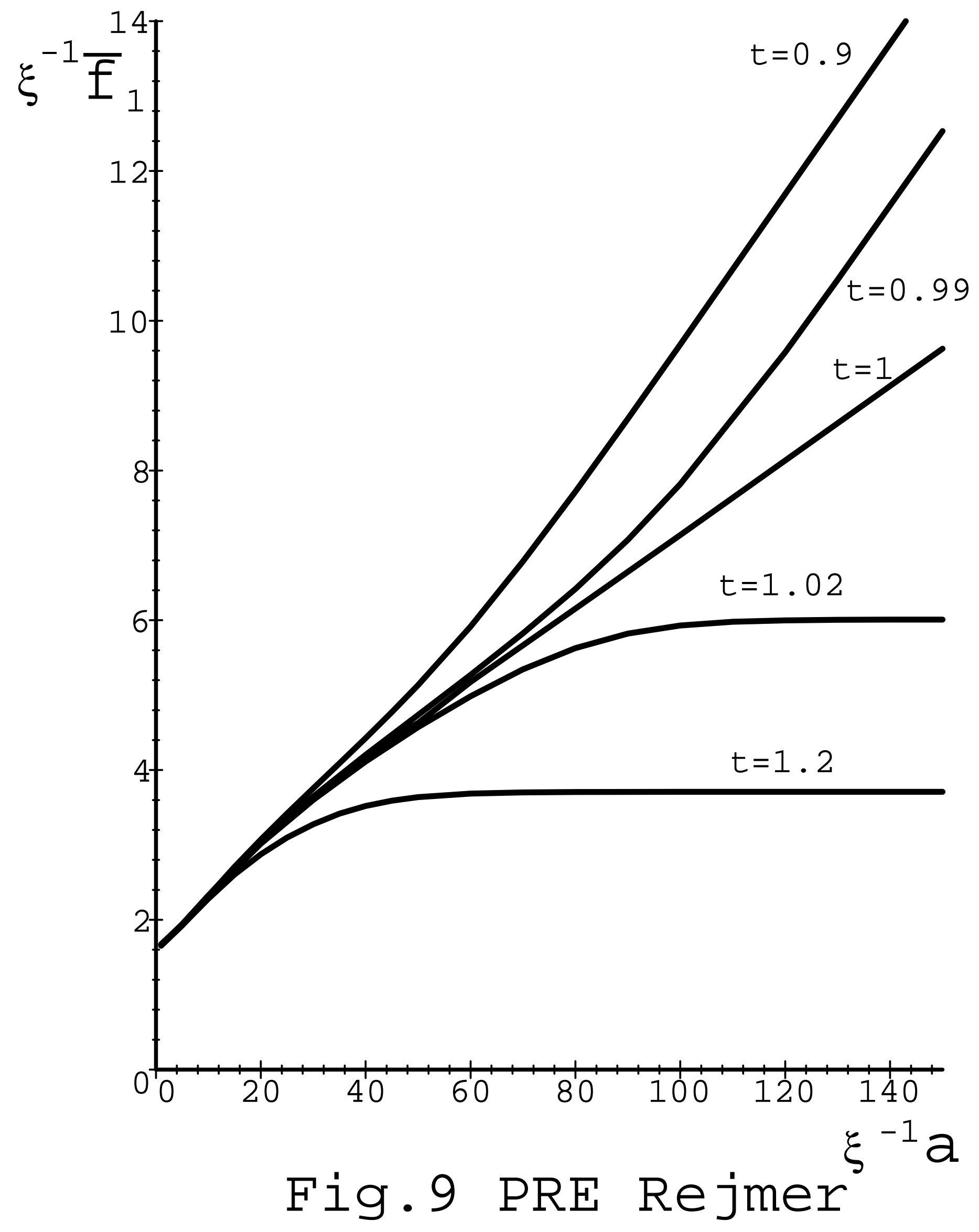

